\title{
Influence of the dispersion environment nature on photoluminescence properties of CdTe nanocrystals in colloidal solutions
}

\author{
O.A. Kapush ${ }^{1}$, S.M. Kalytchuk ${ }^{1}$, L.I. Trishchuk ${ }^{1}$, V.M. Tomashyk ${ }^{1}$, Z.F. Tomashyk ${ }^{1}$, \\ A.S. Kravtsova ${ }^{1}$, D.V. Korbutyak ${ }^{1}$, S.D. Boruk ${ }^{2}$ \\ ${ }^{1}$ V. Lashkaryov Institute of Semiconductor Physics, NAS of Ukraine, 41, prospect Nauky, 03680 Kyiv, Ukraine, \\ Phone: 38(044)525-57-55 ; e-mail: savchuk-olja@rambler.ru \\ ${ }^{2}$ Yuriy Fedkovych Chernivtsi National University, \\ 25, Lesya Ukrainka str., Chernivtsi 58000, Ukraine
}

\begin{abstract}
The physico-chemical properties of low-dimensional structures based on CdTe obtained in the course of colloidal synthesis have been investigated. Analyzed have been the main photoluminescence characteristics of CdTe nanocrystals, which are stabilized by thioglycolic acid and obtained using the deionized water and aqueous solutions of ethylene glycol and glycerol with different concentrations as dispersion environment. It has been shown that stability of colloidal solutions of CdTe nanocrystals depends on the nature of dispersion environment and concentration of stabilizer.
\end{abstract}

Keywords: cadmium telluride, nanocrystal, glycerine, ethylene glycol, glycerol, photoluminescence.

Manuscript received 11.06.12; revised version received 20.06.12; accepted for publication 10.09.12; published online 25.09.12.

\section{Introduction}

Currently, there are many different methods to obtain colloidal nanocrystals (NCs) for their use in various spheres of science and technology. Physical methods provide a high degree of controlling the system parameters, but they are rather complicated in implementation and require cumbersome and valuable equipment.

Chemical methods are simple and economical, more profitable than other methods of synthesis, they allow to obtain CdTe NCs of small sizes, even down to several nanometres.

According to literature data, the most simple and accessible methods of CdTe NCs preparation is the liquid-phase methods of synthesis, in particular - a reverse micelle and colloidal synthesis [1-3]. They offer great opportunities to obtain and study the properties of low-dimensional systems based on CdTe. It is possible to influence not only the size of NCs, but also their shape and structure by changing the synthesis conditions. However, it is necessary to explain the factors that determine the size, structure and shape of CdTe NCs during their colloidal synthesis. In methodologies presented in literature, during colloidal synthesis of CdTe NCs a number of substances that have thio-group are mainly used as the surface stabilizers, but deionized water or dimethylformamide - as a dispersion environment. The role of dispersion environment in the colloidal synthesis of CdTe NCs is related with the nature of solvation processes of interaction between all the participants - initial, intermediate and final reaction products. The redistribution of electron density occurs in the desolvation processes, which in its turn makes a significant effect on interaction processes between the particles in the reaction environment. The solvation of the dissolved particle $\left(\mathrm{CdI}_{2}\right.$, stabilizer $)$ is accompanied by changes in electron structures both of particles and molecules of dispersion environment (deionized water, glycerol, ethylene glycol), and it results in the decrease of enthalpy and entropy of the system. The Gibbs energy becomes lower during transition of the molecules in the solution and its following solvation. If the heat of solute interactions with the solvent is higher, the solubility of 
substances under the same conditions is higher, too. Some solvates, including hydrates and alcoholates of inorganic substances [4], are characterized by a rather high stability (enthalpy of solvation may reach the binding energy value, which is $100-400 \mathrm{~kJ} / \mathrm{mol}$ ). Initial substances as well as intermediate products of the interaction (for example, the complexes of cadmium with stabilizers) and final reaction products (CdTe NCs) are involved in the process of solvation. Thus, solvation is considerably affected by reactivity of the particles.

The energy, which is sufficient to destroy the solvation shell of reacting molecules, is required to hold the reaction of CdTe formation. Solvation of the intermediate and final products may give a gain in the energy, which stimulates the reaction. Thus, the nature of dispersion environment plays a major role in formation of CdTe NCs during their synthesis.

The aim of this work is to study the processes of CdTe NCs formation by using glycerol and ethylene glycol solutions as dispersion environment in colloidal synthesis.

\section{Experimental}

In this work, we have investigated the properties of CdTe dispersions prepared in accord with the methods previously described in $[5,6]$ and developed in $[7,8]$, which involve some significant changes described below. To synthesize colloidal CdTe NCs, a 500-ml flask equipped with barriers, valves, thermometer and electromagnetic stirrer was used as a reactor (Fig. 1). Aqueous solution of $40 \mathrm{ml} 0.1 \mathrm{M} \mathrm{CdI}_{2}$ and $2-15 \mathrm{mmol}$ stabilizer was placed in the reactor to synthesize CdTe NCs. Further, we increased the $p H$ up to the desired value by introducing aqueous solution of $1.25 \mathrm{M} \mathrm{NaOH}$ into the mixture; if it was necessary, then the overall volume of the solution $(250 \mathrm{ml})$ was filled by adding a solvent. Deionized water, aqueous solutions of $10-50 \%$ ethylene glycol and 5-25\% glycerol were used as a solvent. To stabilize the surface of CdTe NCs during the synthesis, thioglycol acid $\mathrm{S}\left(\mathrm{CH}_{2} \mathrm{CO}_{2} \mathrm{H}\right)_{2}(99 \%)$ was used. All chemical reagents were used without additional purification, and deionized water with resistivity 2.5 MOhm was taken for preparation of solutions.

According to the results [9], the use of methanol and ethanol as dispersion environment allows to obtain the CdTe dispersions, which are more stable in time than aqueous dispersions. The possibility of the CdTe NCs synthesis in waterless methanol by using $\mathrm{Na}_{2} \mathrm{Te}$ as a source of $\mathrm{Te}^{2-}$ ions is also described in [10]. But the synthesis of CdTe NCs by using methyl or other monatomic alcohols failed. Due to its insolubility, $\mathrm{H}_{2} \mathrm{Te}$ in all monatomic alcohols of the above series passes through the reaction medium without entering the chemical reaction with cadmium ions. Then it almost completely gets the exhaust gases settings and is neutralized in $0.1 \mathrm{M}$ solution of sodium hydroxide, forming a black sediment of dispersed Te.

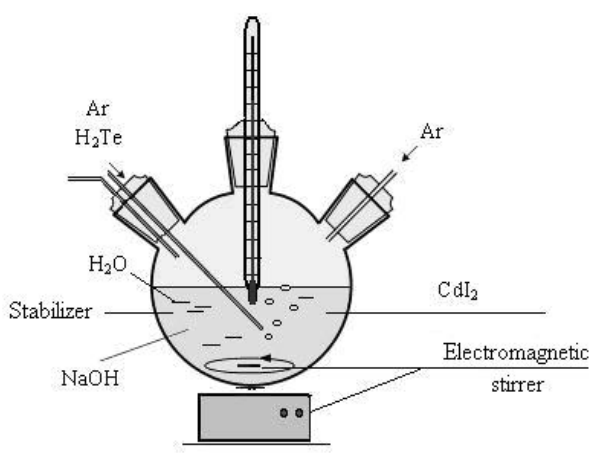

Fig. 1. The setup for CdTe NCs synthesis.

Investigations of optical properties of the solutions were performed in silica and polystyrene cuvettes, using dispersion environment for comparison (deionized water and aqueous solutions of glycerol and ethylene glycol). The photoluminescence (PL) spectra were measured at room temperature, with PL excitation performed using a $\mathrm{He}-\mathrm{Cd}$ laser with the wavelength $325.0 \mathrm{~nm}$ and power $10 \mathrm{~mW}$. Registration of PL signal was carried out using a plant based on MDR-23 spectrometer equipped with uncooled photomultiplier FEU-100 with computer control scanning of the spectrum.

\section{Results and discussions}

The researches have shown that the nature of dispersion environment has a significant influence on the processes of CdTe NCs formation during their colloidal synthesis. The evidence of this is that when synthesizing CdTe $\mathrm{NCs}$ in deionized water with further adding some quantity of ethylene glycol or glycerol into the solution, the changes in optical properties of the solutions are not observed. There are changes in optical properties of the obtained cadmium telluride dispersions and their stability in time, when ethylene glycol and glycerol are present in the reaction environment during the synthesis. The dependence of changes in the properties of the obtained CdTe NCs solutions on the concentration of glycerol and ethylene glycol in the reaction environment was observed (Fig. 2).

As seen from Fig. 2, the use of glycerol and ethylene glycol solutions as dispersion environment during the synthesis allows us to obtain colloidal solutions; the PL bands are characterized by a significant blue shift in comparison with the corresponding aqueous solutions. The characteristic value of full width at half height (FWHH) of PL line for CdTe NCs is about $200 \mathrm{meV}(45 \mathrm{~nm})$ and it is indicative of narrow size distribution of NCs ( $\pm 10 \%$ ). Fig. 2b (curves 2 and 3 ) shows that increase of the glycerol concentration leads to broadening of the spectral line, its gradual red shift and the appearance of some tightening of the long-wave edge at $25 \%$ concentration. It suggests the change in size of CdTe NCs, which are formed, and the change in 
dispersion degree of solution. The use of ethylene glycol (Fig. 2c) as dispersion environment does not lead to a similar effect. The width of spectral lines remains practically the same for the whole range of concentrations. A gradual displacement of the spectrum is observed (Fig. 2c) with increasing the ethylene glycol concentration from $10 \%$ up to $50 \%$. Probably, this is
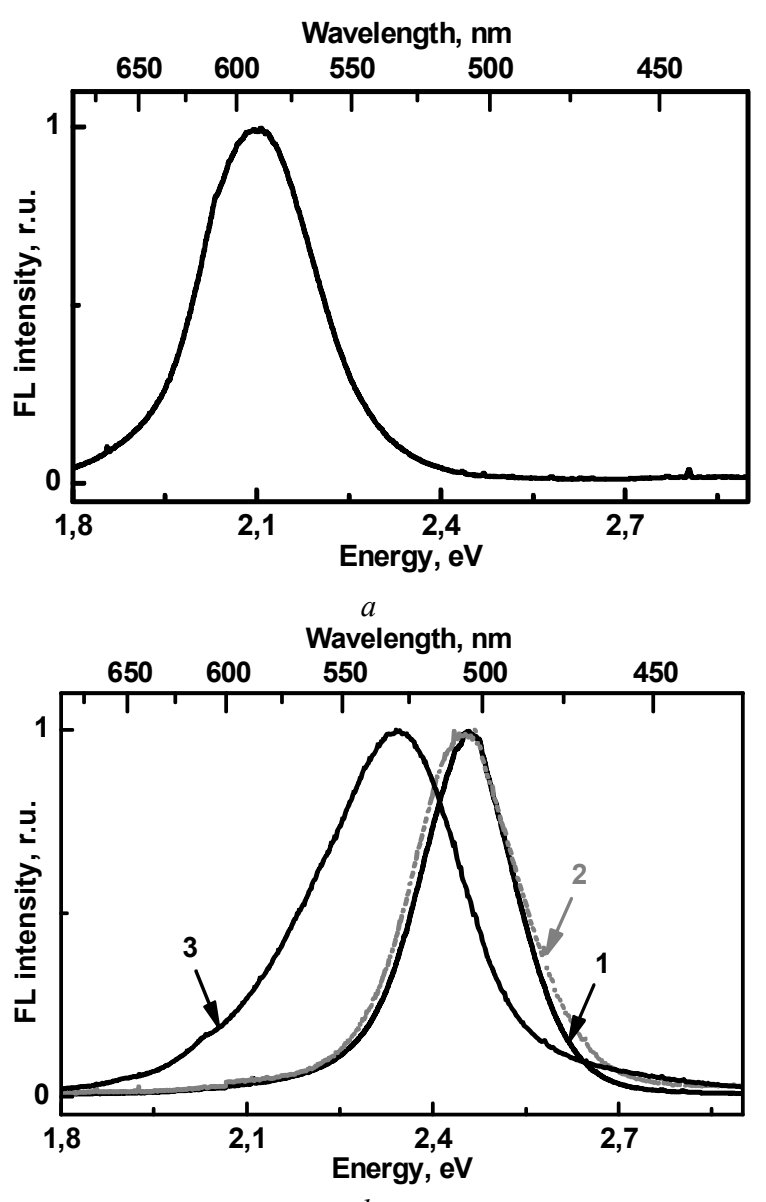

$b$

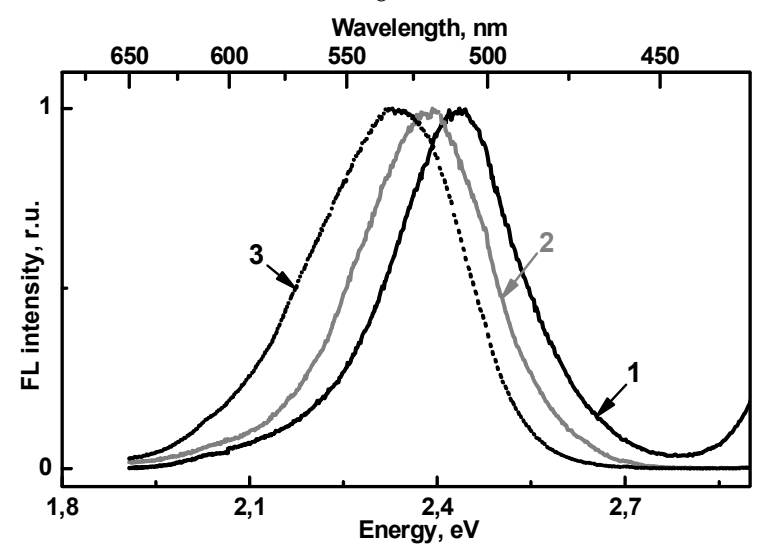

$c$

Fig. 2. The PL spectra of CdTe NCs colloidal solutions obtained using water $(a)$, glycerol solutions $(b)$ with the concentrations 5(1), 10(2), 25(3), and ethylene glycol solutions $(c)$ with the concentrations $10(1), 25(2)$, $50 \%(3)$ as a dispersion environment. due to the fact that the increase in molecular weight solvent and viscosity of the environment in comparison with water leads to the increase of inertia in the growth processes of NCs germs. As a result, the particles are formed with slight variation in size. The hydroxyl groups of ethylene glycol and glycerol can also compete with TGA molecules in the process of modifying the surface of CdTe NCs, which grow, thereby creating a dense isolating layer around the particles and increasing a stabilization degree due to electrical and steric factors. However, the excessively high concentration of ethylene glycol and glycerol may lead to the decrease of nucleation rate and, consequently, the system will be formed from larger NCs. Thus, the stability of these systems in time will be significantly reduced.

It has been found that during the synthesis of CdTe NCs the most optimal concentration of ethylene glycol and glycerol in the reaction environment is $10 \%$. The saving cadmium telluride dispersion, which is obtained under these conditions for 6 months, doesn't lead to significant changes in their optical properties; there is only a small spectral diffusion. In the solutions containing $5 \%$ glycerol, a white precipitate of unknown nature appears with time. All the solutions with the concentration of ethylene glycol and glycerol over $10 \%$ are characterized by coagulation of CdTe NCs, which is observed in a few days. For several months, there is a gradual illumination of colloidal solution, and aggregates precipitate, which is more intensive when the concentration of cadmium telluride in the solution is higher. This is a direct evidence of forming the larger aggregates of CdTe NCs.

\section{Conclusions}

It has been shown that the nature of dispersion environment significantly effects on the formation processes of $\mathrm{CdTe} \mathrm{NCs}$, which are stabilized by thioglycolic acid during their colloidal synthesis. In particular, it has been ascertained that using the dispersion environment of alcohols with two and three $\mathrm{OH}^{-}$groups can significantly increase the sedimentation stability of these dispersions.

It has been shown that the increase in environment viscosity, using aqueous solutions of glycerol and ethylene glycol as dispersion environment in the colloidal synthesis of CdTe NCs, can increase stability of solutions and PL intensity. Also, ethylene glycol and glycerol molecules can compete with thioglycolic acid molecules in the stabilization processes of CdTe NCs.

The most optimal concentration of ethylene glycol and glycerol in the reaction environment has been found to be $10 \%$.

\section{References}

1. A.L. Rogach, Nanocrystalline CdTe and $\mathrm{CdTe}(\mathrm{S})$ particles: wet chemical preparation, size-dependent 
optical properties and perspectives of optoelectronic applications // Mater. Sci. and Eng. B. 69(3), p. 435-440 (2000).

2. D. Ingert, M.P. Pileni, Limitations in producing nanocrystals using reverse micelles as nanoreactors // Adv. Funct. Mater. 11(2), p. 136-139 (2001).

3. N. Gaponik, D.V. Talapin, A.L. Rogach et al., Thiol-capping of CdTe nanocrystals: an alternative to organometallic synthetic routes // J. Phys. Chem. $B$, 106(29), p. 7177-7185 (2002).

4. V.V. Moskva, Solvents in organic chemistry // Sorosovskii Obrazovatelnyi Zhurnal, 4, p. 44-50 (1999), in Russian.

5. T. Rajh, O.I. Micic, A.J. Nosik, Synthesis and characterization of surface-modified colloidal cadmium telluride quantum dots // J. Phys. Chem. 97(46), p. 11999-12003 (1993).

6. C.B. Murray, D.J. Norris, M.G. Bawendi, Synthesis and characterization of nearly monodisperse $\mathrm{CdE}$ $(\mathrm{E}=\mathrm{S}, \mathrm{Se}, \mathrm{Te})$ semiconductor nanocrystallites // J. Am. Chem. Soc. 115 (19), p. 8706-8715 (1993).
7. J. Duan, L. Song, J. Zhan, One-pot synthesis of highly luminescent CdTe quantum dots by microwave irradiation reduction and their $\mathrm{Hg}^{2+}$ sensitive properties // Nano Res. 2(1), p. 61-68 (2009).

8. A.L. Rogach, T. Franzl, T.A. Klar et al., Aqueous synthesis of thiol-capped CdTe nanocrystals: stateof-the-art // J. Phys. Chem. C. 111(40), p. 1462814637 (2007).

9. O.A. Savchuk, S.D. Boruk, S.G. Dremlyuzhenko, V.N. Tomashik, I.N. Yurijchuk, Influence of acids that contain phosphorus on stability of suspensions of finely dispersed powders cadmium of telluride // Scientific Notes of Taurida V. Vernadsky National University. - Series: Biology, chemistry. 23(62), N. 4, p. 294-300 (2010).

10. D.L. Schulz, M. Pehnt, D.H. Rose et al., CdTe thin films from nanoparticle precursors by spray deposition // Chem. Mater. 9(4), p. 889-900 (1997). 